\title{
Pion photoproduction on the nucleon
}

\author{
Haiyan $\mathrm{Gao}^{1}$, Lingyan Zhu ${ }^{2}$ \\ ${ }^{1}$ Triangle Universities Nuclear Laboratory and the Department of Physics, Duke University, \\ Durham, North Carolina, U.S.A. \\ ${ }^{2}$ University of Illinois, Urbana-Champaign, Urbana, IL, U.S.A. \\ for the Jefferson Lab E94-104 and E02-010 Collaboration
}

\begin{abstract}
The $\gamma n \rightarrow \pi^{-} p$ and $\gamma p \rightarrow \pi^{+} n$ reactions are essential probes of the transition from meson-nucleon degrees of freedom to quark-gluon degrees of freedom in exclusive processes. The cross sections of these processes are also advantageous, for the investigation of oscillatory behavior around the quark counting prediction, since they decrease relatively slower with energy compared with other photon-induced processes. In this talk, we discuss recent results on the $\gamma p \rightarrow \pi^{+} n$ and $\gamma n \rightarrow \pi^{-} p$ processes from Jefferson Lab experiment E94-104. We also discuss a new experiment in which singles $\gamma p \rightarrow \pi^{+} n$ measurement from hydrogen, and coincidence $\gamma n \rightarrow \pi^{-} p$ measurements at the quasifree kinematics from deuterium for center-of-mass energies between $2.3 \mathrm{GeV}$ to $3.4 \mathrm{GeV}$ in fine steps at a center-of-mass angle of $90^{\circ}$ are planned. The proposed measurement will allow a detailed investigation of the oscillatory scaling behavior in photopion production processes.
\end{abstract}

\section{INTRODUCTION}

Exclusive processes are essential to studies of transitions from the non-perturbative to the perturbative regime of Quantum Chromodynamics (QCD). The differential crosssection for many exclusive reactions [1] at high energy and large momentum transfer appear to obey the quark counting rule [2]. The constituent counting rule predicts the energy dependence of the differential cross section at fixed center-of-mass angle for an exclusive two-body reaction at high energy and large momentum transfer as follows:

$$
d \sigma / d t=h\left(\theta_{c m}\right) / s^{n-2},
$$

where $s$ and $t$ are the Mandelstam variables, $s$ is the square of the total energy in the center-of-mass frame and $t$ is the momentum transfer squared in the $s$ channel. The quantity $n$ is the total number of elementary fields in the initial and final states, while $h\left(\theta_{c m}\right)$ depends on details of the dynamics of the process. The quark counting rule was originally obtained based on dimensional analysis of typical renormalizable theories. The same rule was later obtained in a short-distance perturbative QCD approach by Brodsky and Lepage[3]. Despite many successes, a model-independent test of the approach, called the hadron helicity conservation rule, tends not to agree with data in the similar energy and momentum region. The presence of helicity-violating amplitudes indicates that the short-distance expansion cannot be the whole story. In addition some of the cross-section data can also be explained in terms of non-perturbative calculations [4].

In recent years, a renewed trend has been observed in deuteron photo-disintegration experiments at SLAC and JLab [5, 6, 7]. Onset of the scaling behavior has been observed 
in deuteron photo-disintegration [5, 6, 7] at a surprisingly low momentum transfer of $1.0(\mathrm{GeV} / \mathrm{c})^{2}$ to the nucleon involved. However, a recent polarization measurement on deuteron photo-disintegration [8] shows disagreement with hadron helicity conservation in the same kinematic region where the quark counting behavior is apparently observed. These paradoxes make it essential to understand the exact mechanism governing the early onset of scaling behavior.

The elastic proton-proton $(p p)$ scattering data at high energy and large momentum transfer have shown very interesting characteristics: the scaled $90^{\circ}$ center-of-mass angle data, $s^{10} \frac{d \sigma}{d t}$ show substantial oscillations about the power law behavior. One theoretical interpretation for such oscillatory behavior and the striking spin-correlation [9] in $p p$ scattering involves interference between hard pQCD short-distance and long-distance (Landshoff) [10] amplitudes [11] along with an energy dependent phase arising from gluonic radiative corrections. This effect is believed to be analogous to the Coulombnuclear interference that is observed in low-energy charged-particle scattering. Brodsky and de Teramond [12] suggest that the structure seen in $s^{10} \frac{d \sigma}{d t}(p p \rightarrow p p)$ and the large spin correlations can be attributed to $c \bar{c} u u d u u d$ resonant states. The opening of this channel gives rise to an amplitude with a phase shift similar to that predicted for gluonic radiative corrections. Deviations (oscillations) from the pQCD counting rule above the resonance region has recently been shown in a model of a composite system with two spinless charged constituents [13], employing the so-called concept of "restricted locality" of quark-hadron duality [14].

$\mathrm{Ji}, \mathrm{Ma}$ and Yuan [15] derived a generalized counting rule for exclusive processes at fixed angles involving parton orbital angular momentum and hadron helicity flip. This generalized counting rule opens a new window for probing the quark orbital angular momentum inside the nucleon. Recently, a non-perturbative derivation of generalized counting rules including the orbital angular momentum was obtained by Brodsky and de Teramond [16]. Therefore, it is very important to investigate the scaling behavior in details and to search for QCD oscillations. The photo-pion production reactions are well suited for these purposes because the cross section of these processes decrease relatively slower with energy $\left(\frac{d s}{d t} \propto s^{-7}\right)$ compared with other photon-induced processes. Rough power-law dependence of meson photoproduction seems to agree with the constituent quark counting rule prediction [17] within experimental uncertainties, for example in the case of the $\gamma p \rightarrow \pi^{+} n$ process at a center-of-mass angle of $90^{\circ}$. Yet it is not clear whether the counting rule scaling behavior has been observed in the $\gamma p \rightarrow \pi^{0} p$ process because discrepancies exist between different measurements. For the $\gamma n \rightarrow \pi^{-} p$ process, no cross section data exist above a photon energy of $2.0 \mathrm{GeV}$ prior to the recent Jefferson Lab experiment E94-104 [18].

\section{JEFFERSON LAB EXPERIMENT E94-104}

Experiment E94-104 was carried out in Hall A [19] at the Thomas Jefferson National Accelerator Facility (JLab). The continuous electron beam, at a current around $30 \mu \mathrm{A}$ and energies from 1.1 to $5.5 \mathrm{GeV}$, impinged on a $6 \%$ copper radiator and generated an untagged bremsstrahlung photon beam. The production data were taken with the 15 
cm cryogenic liquid hydrogen (LH2) target for singles $p\left(\gamma, \pi^{+}\right) n$ measurement, or with the liquid deuterium (LD2) target for coincidence $d\left(\gamma, \pi^{-} p\right) p$ measurement. The two High Resolution Spectrometers (HRS) in Hall A were used to detect the outgoing pions and recoil protons. Two new aerogel Čerenkov detectors in the left spectrometer were constructed for this experiment to provide particle identification for positive particles, mainly pions and protons, since the time-of-flight technique fails at high momentum. Details of the Hall A spectrometers can be found [19].

Based on two-body kinematics, the incident photon energy was reconstructed from final states, i.e. the momentum and angle of the $\pi^{+}$in the singles measurement, momenta and angles of the $\pi^{-}$and $p$ in the coincidence measurement. A $100 \mathrm{MeV}$ bin with the center of the bin $75 \mathrm{MeV}$ from the beam energy, was chosen for the data analysis, where the multi-pion contribution was negligible. The data after background subtraction, with cuts on trigger type, coincidence timing, PID (particle identification), acceptance and photon energy, were compared to a modified Monte Carlo simulation code for this experiment based on MCEEP [20] with the same cuts on acceptance and photon energy. The raw cross section was extracted by comparing data and simulation. The distributions of acceptance, reconstructed momentum and photon energy were in good agreement with results obtained from simulations. Details on the simulation and the bremsstrahlung photon flux calculation can be found [21].

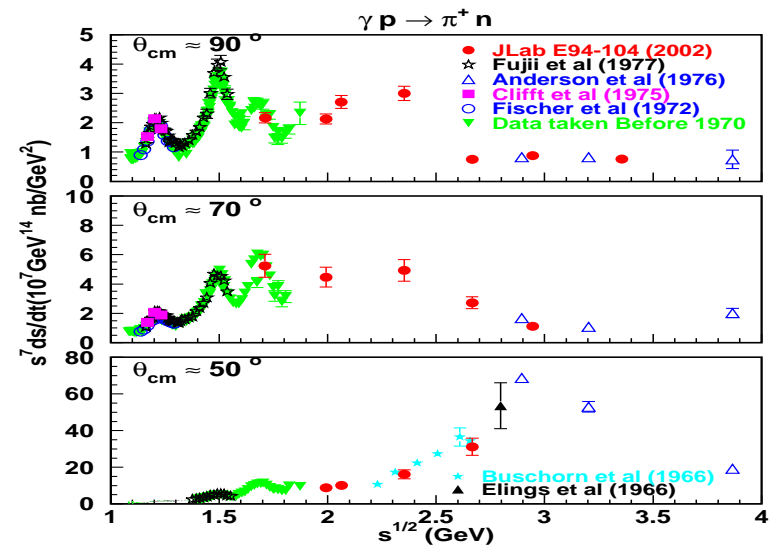

FIGURE 1. The scaled differential cross section $s^{7} \frac{d \sigma}{d t}$ versus center-of-mass energy for the $\gamma p \rightarrow \pi^{+} n$ at $\theta_{c m}=90^{\circ}, 70^{\circ}, 50^{\circ}$.

Important correction factors were applied to deduce the final cross section such as the nuclear transparency factor in the deuteron due to the final state interaction, material absorption of pions and protons, pion decay loss, detection efficiency, etc.. Analysis details can be found in [21,22]. The total errors were dominated by systematic errors and were estimated to be $10 \%$ in cross section, while point-to-point uncertainties for the three kinematics at 3.3,4.2 and $5.5 \mathrm{GeV}$ to be $5 \%$. The statistical errors were approximately $2 \%$. The results [23], as shown in Figure 1 and Figure 2, agree with the world data within uncertainties in the overlapping region. The data at $\theta_{c m}=70^{\circ}, 90^{\circ}$ exhibit a global scaling behavior predicted by the constituent counting rule in $\pi^{-}$channel, similar to what was observed in the $\pi^{+}$channel at similar center-of-mass angles. The data at $\theta_{c m}=50^{\circ}$ do not display scaling behavior and may require higher photon energies for the observation of the onset of the scaling behavior. The data suggest that a transverse 


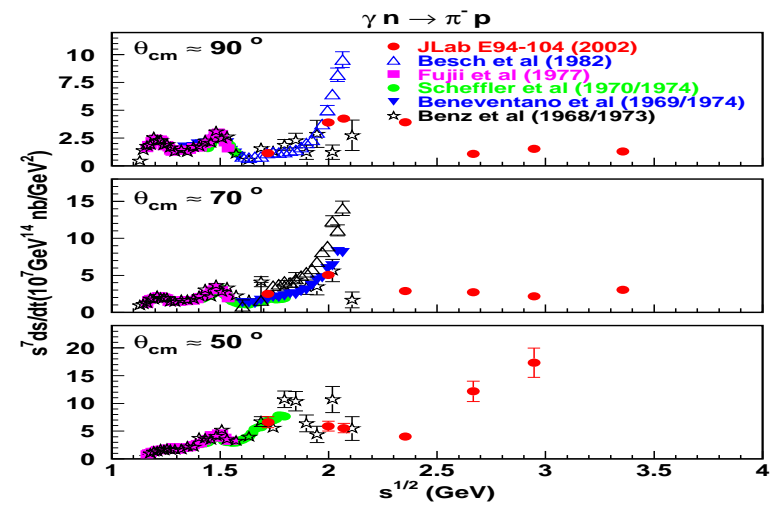

FIGURE 2. The scaled differential cross section $s^{7} \frac{d \sigma}{d t}$ versus center-of-mass energy for the $\gamma n \rightarrow \pi^{-} p$ at $\theta_{c m}=90^{\circ}, 70^{\circ}, 50^{\circ}$.

momentum of around $1.2 \mathrm{GeV} / \mathrm{c}$ might be the scale governing the onset of scaling for the photo-pion production, which is consistent with what has been observed in deuteron photodisintegration [6]. Data in these two channels at $90^{\circ}$ show possible oscillations around the scaling behavior in similar ways as suggested by the insets in Fig. 3 and Fig. 4. Note that this possible oscillatory behavior occurs above the known baryon resonance region. Unfortunately, the coarse and few photon energy settings of this experiment do not allow us to claim the observation of oscillations. Measurements with much finer binning, planned at JLab [29], are essential for the confirmation of such oscillatory scaling behavior.

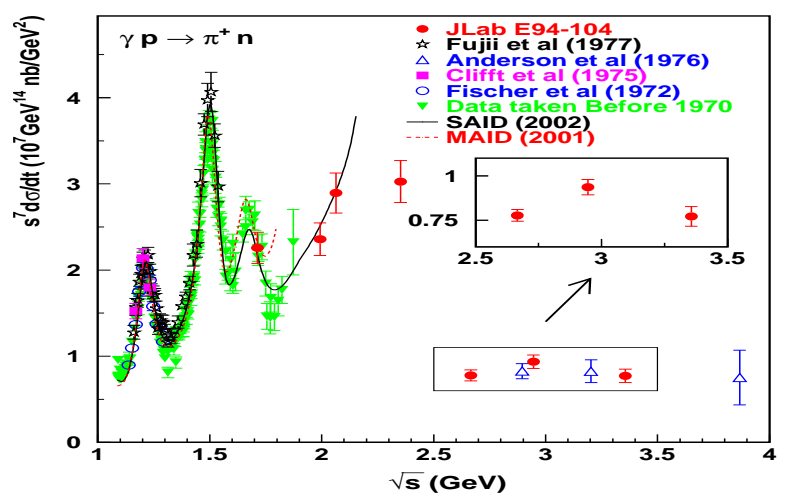

FIGURE 3. The scaled differential cross section $s^{7} \frac{d \sigma}{d t}$ versus center-of-mass energy for the $\gamma p \rightarrow \pi^{+} n$ at $\theta_{c m}=90^{\circ}$. The data from JLab E94-104 are shown as solid circles. The error bars for the new data include statistical and systematic uncertainties, except those in the inset in which only point-to-point systematic uncertainties are included to highlight the possible oscillatory scaling behavior.

\section{JEFFERSON LAB EXPERIMENT E02-010}

Recently a new experiment [29] was approved to carry out a measurement of the photopion production cross-section for the fundamental $\gamma n \rightarrow \pi^{-} p$ process from a ${ }^{2} \mathrm{H}$ target 


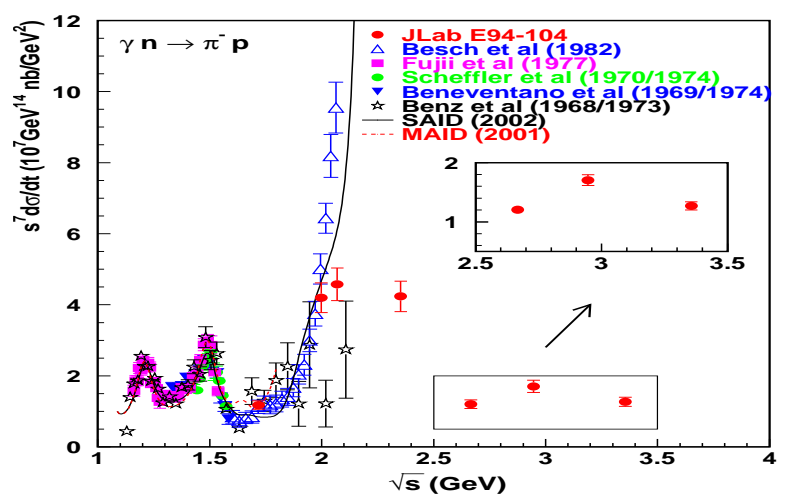

FIGURE 4. The scaled differential cross section $s^{7} \frac{d \sigma}{d t}$ versus center-of-mass energy for the $\gamma n \rightarrow \pi^{-} p$ at $\theta_{c m}=90^{\circ}$. Only point-to-point systematic uncertainties are shown in the inset as in Fig.3.

and for the $\gamma p \rightarrow \pi^{+} n$ process from a hydrogen target at a center-of-mass angle of $90^{\circ}$, at $\sqrt{s} \sim 2.25 \mathrm{GeV}$ to $3.41 \mathrm{GeV}$ in steps of approximately $0.07 \mathrm{GeV}$. The new experiment will make individual cross-section measurement with a $2 \%$ statistical uncertainty and a point-to-point systematic uncertainty of $<3 \%$. Such precision will allow the test of the oscillatory behavior in the scaled fundamental cross section measurement. The proposed experiment will only be possible with the unique JLab capability of high luminosity and such an experiment will be carried out in Hall A at JLab. The projected measurements of E02-010 on the $\gamma n \rightarrow \pi^{-} p$ process at $\theta_{c m}=90^{\circ}$ together with future energy upgraded $12 \mathrm{GeV}$ CEBAF are shown in Fig. 5. Also shown is a fit of the E94-104 data based on a two-component model of Jain and Ralston [30].

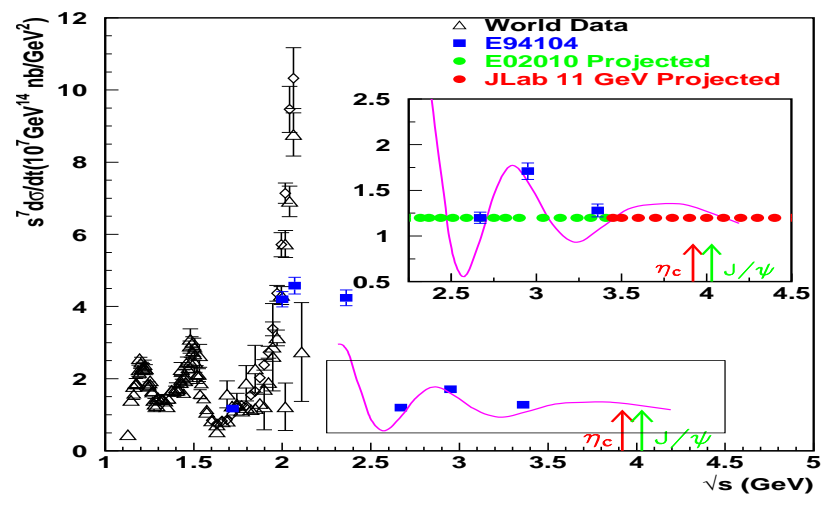

FIGURE 5. The projected measurement from E02-010 and future $12 \mathrm{GeV}$ energy upgrade at JLab on the scaled differential cross section $s^{7} \frac{d \sigma}{d t}$ versus center-of-mass energy for the $\gamma n \rightarrow \pi^{-} p$ at $\theta_{c m}=90^{\circ}$.

\section{ACKNOWLEDGMENTS}

We thank John Arrington and Roy Holt for careful reading of this manuscript, and Dipangkar Dutta in preparing Figure 5. This work is supported by the U.S. Department 
of Energy under contract number DE-FC02-94ER40818 and DE-FG02-03ER41231.

\section{REFERENCES}

1. G. White et al., Phys. Rev. D49, 58 (1994).

2. $\quad$ S.J. Brodsky and G.R. Farrar, Phys. Rev. Lett.31, 1153 (1973); Phys. Rev. D 11, 1309 (1975); V. Matveev et al., Nuovo Cimento Lett. 7, 719 (1973);

3. G.P. Lepage, and S.J. Brodsky, Phys. Rev. D 22, 2157 (1980).

4. $\quad$ N. Isgur and C. Llewelyn-Smith, Phys. Rev. Lett. 52, 1080 (1984).

5. J. Napolitano et al., Phys. Rev. Lett. 61, 2530 (1988); S.J. Freedman et al., Phys. Rev. C 48, 1864 (1993); J.E. Belz et al., Phys. Rev. Lett. 74, 646 (1995); C. Bochna et al., Phys. Rev. Lett. 81, 4576 (1998).

6. $\quad$ E.C. Schulte, et al., Phys. Rev. Lett. 87, 102302 (2001);

7. P. Rossi et al., hep-ph/0405207, submitted to Phys. Rev. Letts.; M. Mirazita et al., Phys. Rev. C 70, 014005 (2004).

8. K. Wijesooriya, et al., Phys. Rev. Lett. 86, , (2)975 (2001).

9. D. G. Crabb et al., Phys. Rev. Lett. 41, 1257 (1978).

10. P. V. Landshoff, Phys. Rev. D 10, 1024 (1974).

11. S.J. Brodsky, C.E. Carlson, and H. Lipkin, Phys. Rev. D 20, 2278 (1979); J.P. Ralston and B. Pire, Phys. Rev. Lett. 61, 1823 (1988); C.E. Carlson, M. Chachkhunashvili, and F. Myhrer, Phys. Rev. D 46, 2891 (1992).

12. S. J. Brodsky, and G. F. de Teramond, Phys. Rev. Lett. 60, 1924 (1988).

13. N. Isgur, S. Jeschonnek, W. Melnitchouk, and J.W. Van Orden, Phys. Rev. D 64, 054005 (2001); F.E. Close and N. Isgur, Phys. Lett. B 509, 81 (2001); S. Jeschonnek and J.W. Van Orden, Phys. Rev. D 65, 094038 (2002); F.E. Close and Q. Zhao, Phys. Rev. D 66, 054001 (2002).

14. Q. Zhao and F. Close, Phys. Rev. Lett. 91, 022004 (2003).

15. X. Ji, J.-P. Ma, F. Yuan, Phys. Rev. Letts. 90, 241601 (2003).

16. S.J. Brodsky, G.F. de Teramond, Phys.Lett.B 582, 211 (2004), also hep-th/0310227

17. R.L. Anderson et al., Phys. Rev. D14, 679 (1976).

18. Jefferson Lab Experiment E94-104, Spokespersons: H. Gao, R.J. Holt.

19. J. Alcorn et al., Nucl. Instrum. Methods A522, 294 (2004).

20. P. Ulmer et al., http://www.physics.odu.edu/ ulmer/mceep/mceep.html.

21. L.Y. Zhu, Ph.D. thesis, February, 2004, Massachusetts Institute of Technology (unpublished).

22. L.Y. Zhu et al., Phys. Rev. Lett. 91, 022003 (2003).

23. L.Y. Zhu et al., nucl-ex/0409018, submitted to Phys. Rev. C.

24. K.-H. Hellwege, Numerical Data and Functional Relationships in Science and Technology, Group I, Volume 8: H. Genzel, P. Joos and W. Pfeil, Photoproduction of Elementary Particles, (1973).

25. HEPDATA: REACTION DATA Database, http://durpdg.dur.ac.uk/hepdata/reac.html.

26. H.-J. Besch et al., Z. Phys. C 16, 1 (1982).

27. I. I. Strakovsky (private communication); R. A. Arndt et al., nucl-th/0205067 (2002), accepted by Phys. Rev. C; http://gwdac.phys.gwu.edu/

28. I. I. Strakovsky (private communication); S. S. Kamalov, et al., Phys. Rev. C 64, 032201 (2001)(A dynamical model); D. Drechsel, et al., Nucl. Phys. A 645, 145 (1999)(a unitary isobar model); http://www.kph.uni-mainz.de/MAID/ MAID2001 refers to the Nov. 2001 version of the MAID solution from S. S. Kamalov.

29. Jefferson Lab Proposal PR02-010, Spokespersons: D. Dutta, H. Gao and R.J. Holt.

30. J.P. Ralston and B. Pire, Phys. Rev. Lett. 61, 1823 (1988); J.P. Ralston and B. Pire, Phys. Rev. Lett. 65, 2343 (1990); P. Jain, private communications. 